\title{
ALFA Detector Control System
}

\author{
Luis Seabra, LIP - Laboratório de Instrumentação e Física Experimental de \\ Partículas, Lisbon, Portugal, on behalf of the ALFA group
}

\begin{abstract}
ALFA (Absolute Luminosity For ATLAS) is one of the sub-detectors of ATLAS (A Toroidal LHC Apparatus). The ALFA system is composed by two stations installed in the LHC tunnel $240 \mathrm{~m}$ away from each side of the ATLAS interaction point. Each station has a vacuum and ventilation system, movement control and all the required electronics for signal processing. The Detector Control System (DCS) provides control and monitoring of several components and ensures the safe operation of the detector contributing to good Data Quality. This paper describes the ALFA DCS system including a detector overview, operation aspects and hardware control through a SCADA system, WinCC OA.
\end{abstract}

Keywords-LHC, ATLAS, ALFA, Detector Control System, WinCC, Finite State Machine

\section{INTRODUCTION}

$\mathrm{T}$ HE discovery at CERN of a new particle compatible with the Higgs boson was an achievement reached by the Large Hadron Collider (LHC) and its experiments, ATLAS and CMS (Compact Muon Solenoid). The ATLAS detector is composed of several sub-detectors, including the ALFA [1] luminosity detector.

ALFA is a forward detector installed in the LHC tunnel $240 m$ away from the ATLAS Interaction Point (IP). The ALFA system consists of four detector stations, two in each side of the IP separated by $4 m$, and each station has two ALFA detectors. Each ALFA detector is inside one Roman Pot that protects it from the LHC high vacuum with a secondary safety vacuum. Figure 1 shows a schematic view of the detector inside the pot and Figure 2 shows the station inside the LHC tunnel.

The detector was built to measure the absolute luminosity of ATLAS, by detection of the elastically scattered protons down to very small angles $(3.5 \mu \mathrm{rad})$ very close to the beam, in the 33 millimeter range.

ALFA is a tracking detector based in scin-35 tillating optical fibers that detect particles 36 through scintillation processes. The light pro- 37 duced in the fibers travels into MultiAnode 38 PhotoMultiplier Tubes (MAPMTs). The $\mathrm{PMT}_{39}$ Front-end, PMF, is mounted on each MAPMT 40 and it provides high voltage and data han-41 dling through a Field-Programmable Gate Ar-42

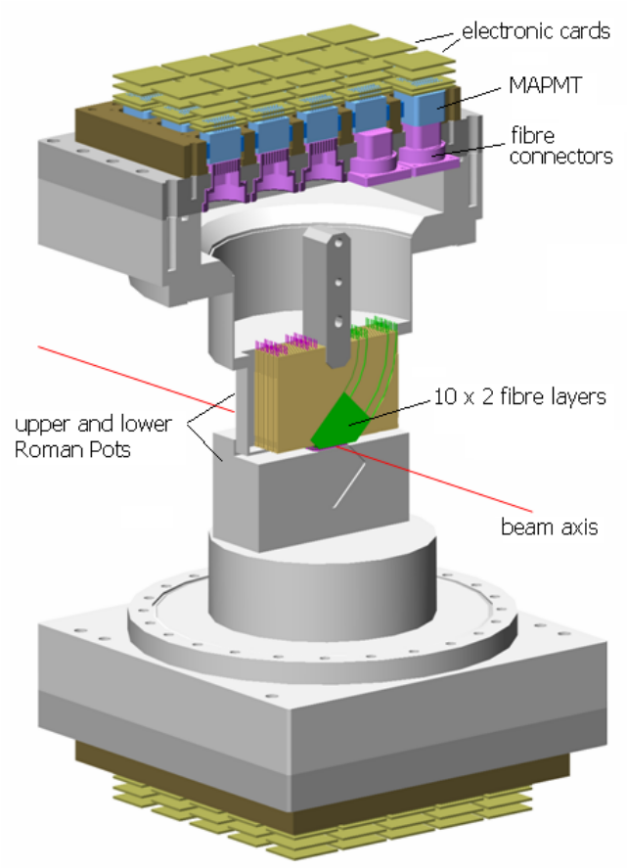

Fig. 1. View of the ALFA detector assembly with the Roman Pot.

ray (FPGA) and a MultiAnode ReadOut Chip (MAROC). The motherboard collects the data from the 23 PMFs and sends it to the Data Acquisition system (DAQ).

The ALFA DCS ensures the monitoring and operation of the detector. Section 2 describes the main DCS systems. Some of these systems are located in the ATLAS electronic cavern (USA15), more than $250 \mathrm{~m}$ from the ALFA stations. 


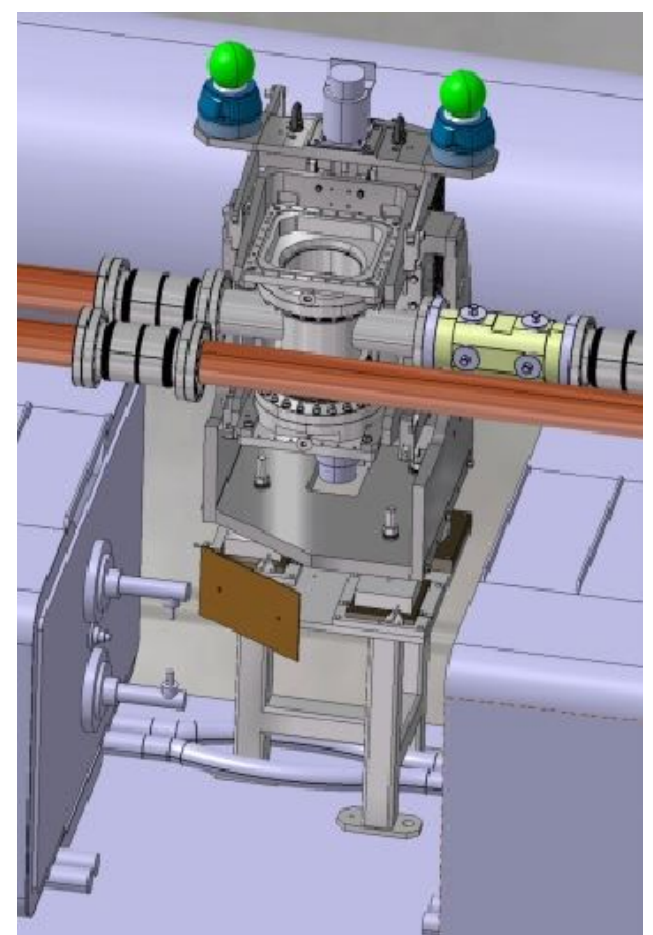

Fig. 2. View of the ALFA station inside the $\mathrm{LHC}_{71}$ tunnel.

\section{The ALFA Main DCS Systems}

\subsection{System Overview}

The Supervisory Control And Data Acquisition 78 (SCADA) is WinCC [2], a commercial package 79 chosen by the Joint Control Project (JCOP) [3] to 80 develop the DCS system for all the LHC exper-81 iments. The JCOP goal is to provide standards 82 for the use of DCS common hardware compo- 83 nents, implementation policies, Back-End (BE) 84 software and operational aspects for all exper- 85 iments. WinCC is a commercial product from 86 SIEMENS, developed to configure, monitor and 87 operate hardware devices and it's described in 88 more detail in Section 3.

The BE system of the ATLAS experiment is 90 organized in three layers as shown is Figure 3.91 The Global Control Stations (GCS) provide 92 overall operations for monitoring and control ${ }_{93}$ of the ATLAS sub-detectors. The Sub-detector 94 Control Station (SCS) is a high-level control 95 of a sub-detector allowing to operate the sub-96 detector in stand-alone mode. Local Control 97 Stations (LCS) are used to handle low level 98 monitoring and control of the Front-End (FE) ${ }_{99}$ hardware. As ALFA is part of the forwardioo

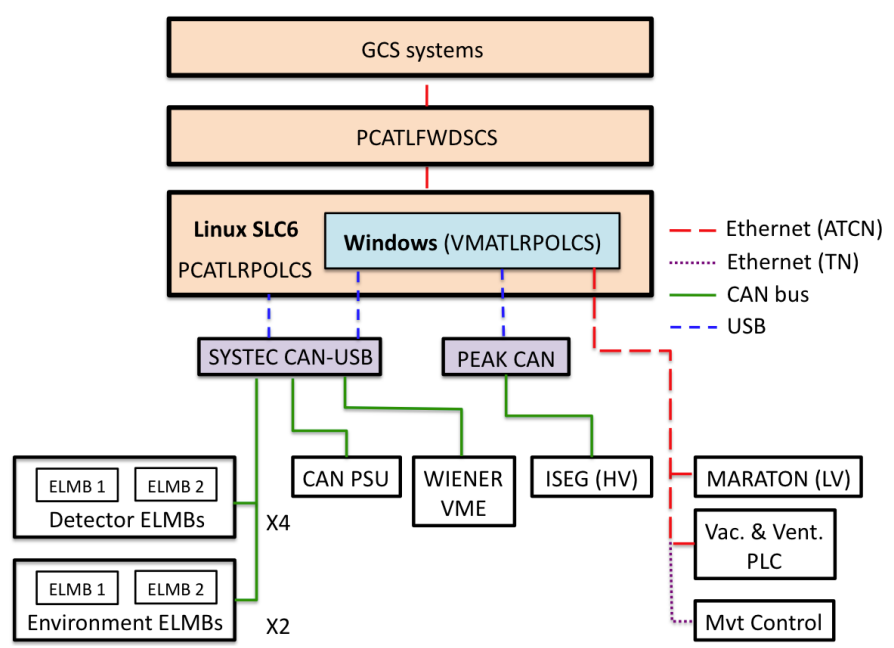

Fig. 3. Hardware architecture in ALFA DCS.

detectors, the LCS is connected to the respective SCS, PCATLFWDSCS (see Figure 3).

The ALFA DCS is hosted in a server (DELL R610) with Linux SLC6 as native operating system. The control interfaces of some commercial components work under Windows operating system so the server hosts also a virtual machine (VM) with Windows Server 2008.

For CAN communication the ALFA DCS uses two CAN-USB interfaces, PEAK and SYSTEC. PEAK is the interface that handles the high voltage $(\mathrm{HV})$ provided by ISEG power supply through an OPC DA (DCOM Acess). This OPC runs only under Windows so the PEAK USB port is connected to the VM. The rest of the CAN communication is handled by SYSTEC that has $8+8$ bus channels, with half of them connected to the native server (Linux) and the other half to the VM. The WIENER VME crate has an OPC DA server which is connected to the SYSTEC part routed to the VM. The other part is used to connect all ELMB [4] (Embedded Local Monitor Boards) related CAN buses where an OPC UA (Unified Architecture) runs under Linux.

The communication for a Maraton crate and a Siemens PLC for vacuum and ventilation is done through OPC DA interfaced by Ethernet. For Movement Control it is also used Ethernet interface but through DIM (Distributed Information Management)[5].

To control and monitor the complete system two SCADA systems under WinCC OA 3.11 
were built. The main system is hosted in the 45 Linux server and it is called ATLRPOLCS (see Figure 3). This project is the core of the ALFA DCS and manages all ELMBs, runs all control ${ }_{148}$ scripts, displays information, contains the FSM $_{149}$ and is the interface for the ATLAS DCS. In the VM there is a scattered project called ATLRPOOPC which operates all OPC clients for the systems with OPC DA.

\subsection{High Voltage Power Supply}

The high voltage is provided by one ISEG $_{157}^{156}$ power supply equipped with 8 modules, one $_{158}$ module per detector. Each module provides high voltage to 27 individual channels for the ${ }_{160}$ MAPMTs and Trigger PMTs, allowing the mon- ${ }_{161}$ itoring of voltages, currents, states $(\mathrm{ON} / \mathrm{OFF})_{162}$ temperatures and alarms.

The communication to the hardware is per- $^{-164}$ formed by an OPC server (isegHVOPCServer) ${ }_{165}$ provided by ISEG. As it was said in Section 2.1 $1_{166}$ this server only runs under Windows so the ${ }_{67}$ software is installed in the VM and the respec ${ }_{168}^{-}$ tive OPC client in the scattered project.

\subsection{Low Voltage Power Supply}

The low voltage system (Maraton) is respon-173 sible to provide low voltage to the front-end $\mathrm{d}_{174}$ electronics (motherboards). This is done by the control and monitoring of two controllers con ${ }_{176}$ nected in the VME crate.

The controllers are interfaced to the DCS by SNMP (Ethernet) and connected to the SCADA by a Wiener OPC server. From this server it's possible to monitor the TCP/IP connection, the $\mathrm{e}^{179}$ crate fans and also to control/monitor voltages ${ }^{180}$ and currents for each channel.

\subsection{VME Crate}

One Wiener VME crate is used in ALFA to host ${ }^{15}$ the Maraton controllers (Section 2.3) and al1 ${ }^{186}$ DAQ related front-end hardware. The crate is ${ }^{187}$ monitored by the DCS via its fan unit using ${ }^{188}$ the same OPC server as the Maraton crates. The server allows to monitor the crate status, ${ }^{189}$ fan speed, temperatures, voltage, current andiso status flags for each channel.

\subsection{ELMB and CAN Power Supply Unit}

In each detector there is one ELMB integrated in the motherboard for monitoring and configuration of the equipment. 28 channels of this ELMB are used for monitoring voltages and currents on the motherboard. It performs a front-end electronics reset (using digital outputs) and also a configuration through SPI (Section 5.2).

There is one ELMB per station for temperature monitoring using PT100 sensors. These sensors are located in different parts of the detector to ensure a safe operation. For Radiation Monitoring it's used one ELMB in one side of the IP (Sector 8-1). A total of 6 radiation sensors are installed at different levels of the detector.

To power the CANbus lines it's used a CAN PSU. It provides the link to the SYSTEC interface and it also powers the digital part of the ELMBs (the analog part is powered by the motherboards).

In total there are 13 ELMBs on the ALFA system connected to the DCS through 7 CAN buses as described in Figure 3. There is one bus line with one ELMB per detector for monitoring and configuration and one bus line with two ELMBs per sector for temperature monitoring, except for Sector 8-1 where the CAN bus line has three ELMBs (two for the temperatures and one for the radiation sensors).

All the ELMBs are connected to the SYSTEC interface where they are readout using the OPC UA that runs under Linux.

\subsection{Vacuum and Ventilation}

The Vacuum and Ventilation system relies on one Siemens programmable logic controller (PLC) for monitoring and control of the aircooling provided by fans and the secondary vacuum, provided by pumps. From the OPC server and client (installed in the VM) it's possible to monitor and control the status (ON/OFF) of the air cooling fan and pumps, close and open valves and select the pumping mode (MANUAL/AUTO).

\subsection{Movement Control}

The monitoring and control of the stations movement along the beam pipe is a standalone 
system fully integrated in the LHC control system. There are two systems involved in the movement control: a National Instruments PXI which is a front-end real time control application and a FESA server that hosts the DIM DNS for the ALFA DCS connection and also interfaces to the CERN Control Control applications.

The ALFA DCS is not the responsible by the stations movement but it has the possibility to publish commands to the PXI for emergency extraction of the pots and movement inhibit commands.

For monitoring purpose are published the PXI/FESA status, switches and stoppers states and the detector positions (motor steps and $\mathrm{a}_{241}$ LVDT).

\section{Detector Control System}

The Detector Control System (DCS) [6] [7] was $_{245}$ built using WinCC OA 3.11 which is a SCADA S24 $_{24}$ base tool. A control station (PC), with WinCC ${ }_{247}$ runs a Project with a number of processes called ${ }_{248}$ Managers. These Managers are responsible for 249 the operation of the Project through sets of in- $\overline{-}_{250}$ structions and can be of different types (Control ${ }_{251}$ scripts, OPC clients, etc). All the DCS data is ${ }_{252}$ stored in objects called Datapoints (DP) and it's $s_{253}$ this data that is processed by the Managers, Data processing is performed in an event-by- ${ }_{255}$ event basis using multi-thread callback routines upon value changes. WinCC is a deviceoriented software allowing to handle a DP as a device and the DP structure, Datapoint Elements (DPE), such as hardware channels of the device (temperatures, currents, etc). From the DPEs it's possible to attach configurations in order to setup alert, archiving, addressing (for hardware), etc.

From the WinCC it's possible to produce graphic interfaces of the system, or parts of it through the use of panels. A Finite State $\mathrm{Ma}_{263}$ chine (FSM) allows to navigate through these panels, calculate states and status of the detec-264 tor and send commands in an easy way for ab65 non expert operator.

\section{Finite State Machine}

The Finite State Machine (FSM) is where the69 operator interacts with the detector for controk ${ }_{70}$ and monitoring.

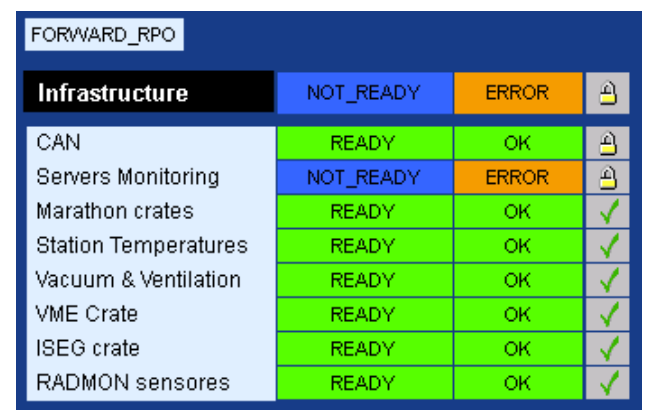

Fig. 4. Infraestructure node of the ALFA DCS, showing the state and status of each one.

The DCS back-end is mapped onto a hierarchy of FSM elements where state changes are propagated upwards and commands downwards in the hierarchy allowing for the operation of the complete detector interacting with a single FSM object at the top of the hierarchy. The top node runs on the GCS, sub-detectors are located on the respective SCS, and all lower level objects are situated on their dedicated LCS connected to the front-end devices. Figure 4 shows the Infrastructure node from the ALFA FSM and the state propagation from the Servers Monitoring node to the Infrastructure.

From the ALFA FSM the operator have the ability to navigate through the detector selecting what he wants to see. Figure 5 shows the ALFA main FSM page where it is available an overview of the system with the beam loss monitors around ALFA stations, position of the beams (BPM) and state of all pots and the infrastructure.

\section{FRONT-END HANDLING}

\subsection{Front-End electronics monitoring and interlock}

The front-end monitoring is performed by a control script that looks into the input channels of the Maraton crate (LV). This control script runs continuously and ensures the protection of the electronics. It looks at all current readout performed locally by the ELMB and will trigger an automatic shutdown of the low voltage if a reading triggers a FATAL alert. 


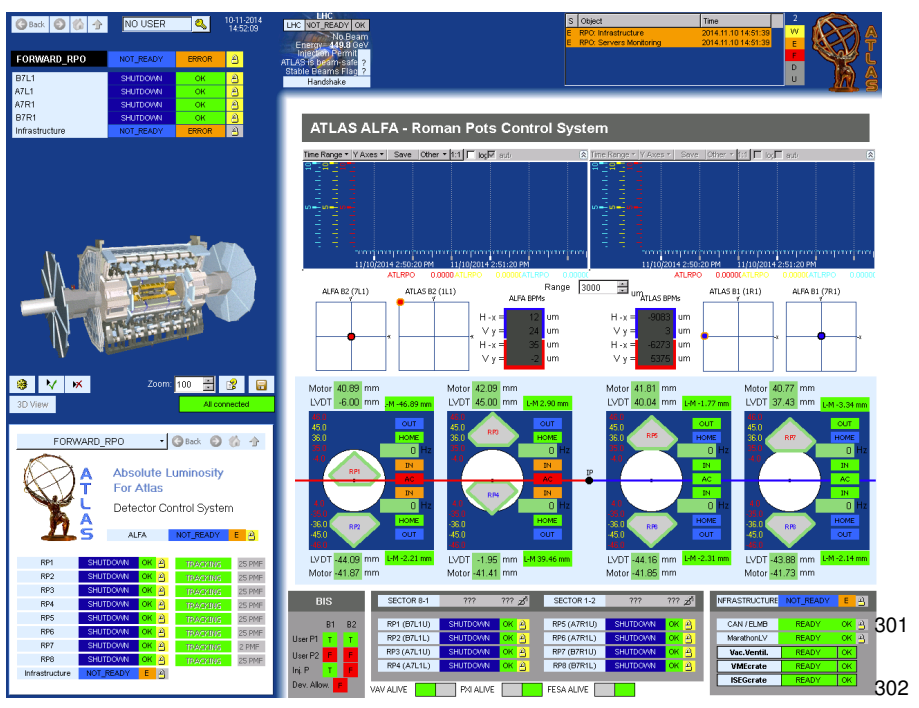

Fig. 5. Main panel of the ALFA DCS from the ${ }^{303}$ FSM point of view.

\subsection{PMF Configuration}

The front-end electronics is configured withio DCS through the SPI interface of the ELMBs 311 For communication between ELMB and FPGAs12 of each detector, a procedure has been designed\$13 for the exchange of 16 bits words. When the 14 ELMB is writing data in the FPGA register i ${ }^{1} 15$ sends 32 bits of data, 16 bits for addressing thes16 register in the FPGA and 16 bits of data. When 17 the ELMB reads data in the FPGA, it sends a 16-bit word giving the register address to be read out and the FPGA returns another 16-bit ${ }^{31}$ word with the register content as an answer. ${ }^{319}$ Using this procedure, the ELMB configures $23^{20}$ FPGAs (one for each PMF) and the two FPGAs ${ }^{321}$ from the trigger mezzanine.

In the PMF configuration procedure the data ${ }^{323}$ sent to the FPGAs is related with the PMF $^{32}$ (gains, thresholds and control bits for each PMF), trigger (pattern, rate and latencies) and LED configuration (pulse width, voltage and ${ }_{226}$ mode).

327

To handle the PMF configuration an user328 interface was developed to set the configura-329 tion parameters. This configuration is activated $d_{30}$ by a FSM command for each pot. During this $\$_{31}$ process a read back test is performed in order ${ }_{32}$ to check for failures in the configuration of all 33 PMFs.

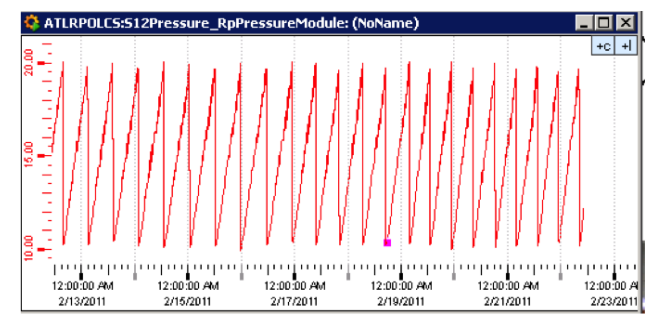

Fig. 6. Pressure trend inside the Roman pot through time. The values were retrieved from Oracle DB using the DCS as a display tool.

\section{DATA HANDLING}

\subsection{Data Archiving}

All relevant data that is related with the detector operation (voltage, currents, etc) is stored in an Oracle online database. This data is stored according to a smoothing mechanism based on value and/or timestamps in order to avoid a huge amount of stored data for parameters that have a low refresh rate or a low value variation in time. Figure 6 shows a pressure trend where it's possible to see the pressure variation in time where the smoothing mechanism was used.

To be available outside the ATLAS technical network the data is stored in an offline database. For physics analysis and data quality some of the online data is also stored in COOL database.

\subsection{Configuration Database (Recipes)}

In order to minimize the risk of operator errors during configuration a recipe mechanism has been developed. This mechanism allows the operator to select configurations schemes accessing a configuration database. These configuration schemes can be related with high voltages, PMF gains, latency, etc.

\subsection{Alert Handling}

The alert handling mechanism of ALFA DCS provides alerts to the operator that can indicate a failure in the hardware or a trigger in a monitored parameter.

An alert can also trigger a notification (email and / or SMS) to the operator. This is done using a control script that checks the status of some sensitive parameters of the detector. 


\section{SUMMARY}

The ALFA DCS includes a large variety of different systems successfully integrated in the ATLAS detector in a coherent way. It was able to provide detector monitoring and safe control during beam operation in the LHC run 1.

\section{ACKNOWLEDGMENTS}

The authors would like to thank the all ALFA community and also the LIP ATLAS group from Lisbon. The work was supported in part by FCT and QREN/COMPETE

\section{REFERENCES}

[1] The ATLAS Collaboration. Detectors for Measurement of Elastic Scattering and Luminosity, CERN/LHCC 2008-04, 2008.

[2] Siemens, WinCC SCADA system, http://w3.siemens.com/mcms/human-machineinterface/en/visualization-software/scada/simaticwincc/Pages/default.aspx

[3] O. Holme, M. Gonzalez Berges, P. Golonka and S. Schmeling, The JCOP framework, proceedings of the $10^{\text {th }}$ International Conference on Accelerator and Large Experimental Physics Control Systems (ICALEPCS 2005), Geneva, Switzerland, 2005.

[4] B. Hallgren, The Embedded Local Monitor Board (ELMB) in the Front-End I/O Control System, $7^{t}$ Workshop for LHC Experiments, Stockholm (2001).

[5] C. Gaspar, M. Dönszelmann and Ph. Charpentier, DIM, a portable, light weight package for information publishing, data transfer and inter-process communication, Comput. Phys. Commun. 140 (2001) 102.

[6] High-Level Triggers, DAQ and DCS Technical Proposal, CERN/LHCC/2000-17 (2000).

[7] A. Barriuso Poy, The detector control system of the ATLAS experiment, 2008 JINST 3 P05006. 